\title{
Die konservative Behandlung der proximalen Oberarmfraktur*
}

$\square$ Christian Krettek, Nael Hawi, Ulrich Wiebking

\section{Zusammenfassung}

Die proximale Humerusfraktur ist eine der häufigsten Frakturen des Menschen. Etwa $80 \%$ der Frakturen sind wenig oder nicht disloziert und damit der konservativen Therapie gut zugänglich, etwa $80 \%$ der Patienten sind über 60 Jahre alt. Winkelstabile Platte und Verriegelungsnagel haben bei hohen Komplikations- und Revisionsraten die Erwartungen vor allem beim älteren Patienten mit Osteoporose nicht erfüllen können. Prospektiv randomisierte Studien aus Finnland und Schweden haben ergeben, dass beim älteren Patienten über 60 Jahre bei der dislozierten 4-Teile-Fraktur die Ergebnisse der Plattenosteosynthese oder der Frakturprothese nicht besser sind als die der konservativen Behandlung. Gleiches gilt auch für die Frakturprothese.

\section{Conservative Treatment of Proximal Humerus Fractures}

Proximal humerus fractures are among the most common fractures. About $80 \%$ of all proximal humerus fractures are undisplaced or minimally displaced and suitable for non-operative treatment. About $80 \%$ of all patients are more than 60 years old. Locked plating and interlocking nailing have not fulfilled the expectations, especially in elderly patients with osteoporotic bone. Prospective randomised studies from Scandinavia have shown that in elderly patients above 60 years of age clinical results and outcome in cases with dislocated threeand four-part fractures treated with locked plates or hemiarthroplasty are not better than those for patients treated non-operatively.

\section{Einleitung}

Die Behandlung dislozierter Frakturen des proximalen Humerus bleibt eine Herausforderung. Frakturtyp, Dislokationsgrad und Alter gelten als wesentliche Prädiktoren für das funktionelle Ergebnis [5]. Für die Behandlung dislozierter Frakturen stehen eine Reihe von Optionen zur Verfügung. Die chirurgischen Behandlungsmöglichkeiten beinhalten offene oder geschlossene Reposition und Stabilisierungsverfahren unter Verwendung von Drähten, Cerclagen, Nägeln, Fixateur externe und Schrauben. Prothetischer Gelenkersatz unter Verwendung konventioneller oder inverser Prothesen oder konservative Therapie sind weitere Möglichkeiten $[1,2,9,10$, $13,14,16]$.

OP-JOURNAL 2013; 29: 32-38

(C) Georg Thieme Verlag KG Stuttgart · New York DOI http://dx.doi.org/10.1055/s-0032-1328483
Plattensysteme mit nicht winkelstabiler Platten-Schrauben-Verbindung sind insbesondere beim Patienten mit schwerer Osteoporose durch eine hohe Repositionsverlustrate kompliziert.

Vor diesem Hintergrund war der Einsatz winkelstabiler Plattenfixateure mit groBen Erwartungen in Bezug auf einen verbesserten Erhalt des Repositionsergebnisses verbunden $[6,15]$. Diese Erwartungen haben sich vor dem Hintergrund hoher Komplikationsraten und enttäuschender funktioneller Ergebnisse nicht erfüllt.

Zwar haben sich die radiologisch-anatomischen Ergebnisse durch den Einsatz

\footnotetext{
* Die Publikation enthält Auszüge aus Krettek C, Wiebking U. Proximale Humerusfraktur: Ist die winkelstabile Plattenosteosynthese der konservativen Behandlung überlegen? Unfallchirurg 2011; 114: 1059-1067 (mit freundlicher Genehmigung des Springer Verlags, Heidelberg).
}

der Winkelstabilität verbessert, die funktionellen Ergebnisse hinken aber hinter der verbesserten Anatomie weit hinterher.

\section{Die Studienlage - 3 prospektiv randomisierte Studien}

\section{Fjalestad-Studie: dislozierte 3/4-Teile-} Frakturen - Platte vs. konservativ

Die norwegische Arbeitsgruppe um Fjalestad publizierte 2011 eine 2-armige, prospektiv randomisierte Studie mit insgesamt 50 Patienten (Mindestalter 60 Jahre) [3] (Tab. 1). Eingeschlossen wurden nur schwere, dislozierte $\left(>45^{\circ}\right.$ oder > $10 \mathrm{~mm}$ ) 3- und 4-Teile-Frakturen der Gruppen 11-B2 $(\mathrm{n}=13$ in beiden Gruppen) und 11-C2 ( $\mathrm{n}=12$ in beiden Gruppen) nach der AO/OTA-Klassifikation (Abb. 1 und 2). 25 Patienten wurden operativ (winkelstabile Platte, Cerclagen, Physiotherapie ab Tag 3), 25 konservativ (geschlossene Reposition, wenn Fragmentverschiebung $>50 \%$ des Schaftdurchmessers, Physiotherapie ab Tag 15) behandelt. Zwei Patienten aus der operierten Gruppe verstarben. Die 12-Monats-Analyse zeigte keine statistisch signifikanten Unterschiede im funktionellen Ergebnis (Constant Score, Patienten Self-Assessment mit dem American Shoulder and Elbow Surgeons Standardized Shoulder Assessment Form) zwischen konservativer und operativer Therapie. Lediglich die radiologischen Scores zeigten erwartungsgemäß Vorteile zugunsten der operativen Therapie.

Die Bedeutung dieser Studie ist hoch. Erstmals gelang damit der Nachweis, dass die konservative Therapie der winkelstabilen Plattenosteosynthese vom funktionellen Ergebnis ebenbürtig ist, bei geringerer Rate an Komplikationen [7].

Die traditionelle Art und Weise, Ergebnisse und Effektivität nach chirurgischer 
Tab. 1 Funktionelle Ergebniss und Komplikationen 1 Jahr nach Fraktur der Studie von Fjalestad et al. [3,4] (aus [8]).

\begin{tabular}{|c|c|c|c|}
\hline & $\begin{array}{l}\text { winkelstabile } \\
\text { Platte }(n=25)\end{array}$ & $\begin{array}{l}\text { konservative Be- } \\
\text { handlung }(n=25)\end{array}$ & \\
\hline AO Typ B2 & 13 & 13 & \\
\hline AO Typ C2 & 12 & 12 & \\
\hline Constant Score (95\%-KI) & $52,3(43,2-61,2)$ & $52,2(44,6-59,7)$ & 0,1 (n.s.) \\
\hline Constant Score Differenz (zwischen verletzt und unverletzt) (SD) & $35,2(17,2)$ & $32,8(16,2)$ & $2,4(p=0,62)$ \\
\hline Adjusted Constant Score (SD) & $74,4(29,4)$ & $74,4(22,9)$ & $0,0(p=0,99)$ \\
\hline ASES Self-Assessment Score (verletzte Seite) (SD) & $14,8(6,6)$ & $15,5(6,9)$ & $0,7(p=0,71)$ \\
\hline verstorbene Patienten & 2 & - & \\
\hline Implantatversagen oder Dislokation, die operative Maßnahmen erfordern & 1 & 1 & \\
\hline Pseudarthrose (schmerzlos) & - & 2 & \\
\hline Schraubenpenetration & 7 & - & \\
\hline Implantentfernung wegen Schraubenpenetration & 3 & - & \\
\hline fortgeschrittene avaskuläre Nekrose & 2 & - & \\
\hline Summe fraktur- oder behandlungsbezogener Komplikationen & $13(52 \%)$ & $3(12 \%)$ & \\
\hline 15D Scores (nach 12 Monaten) & $0,841(0,105)$ & $0,819(0,083)$ & $p=0,436$ \\
\hline Behandlungskosten & $23953 €$ & $21878 €$ & \\
\hline
\end{tabular}



Abb. 1 AO-Klassifikation.

oder konservativer Behandlung am Bewegungsapparat darzustellen, sind Bewegungsumfang, Muskelkraft, sowie radiologische Befunde wie Fragmentposi- tion und Frakturheilung. Die OutcomeForschung hat hier zeigen können, dass objektive Parameter nicht immer mit der subjektiven Einschätzung der Patienten übereinstimmen. 


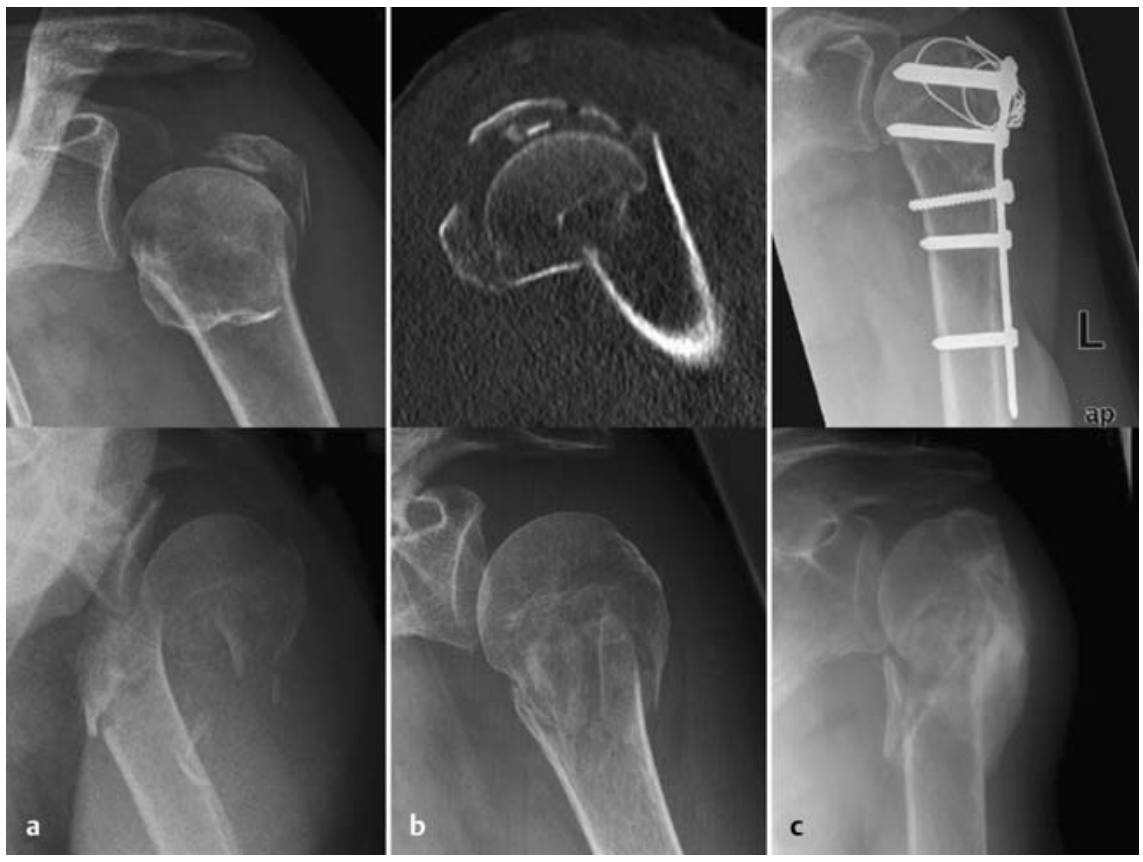

Abb. 2 Oben: operativ behandelte OTA Type 11-C2-Fraktur (intrakapsulär). (a) Konventionelle präoperative a.-p. Aufnahme. (b) Coputertomogramm mit 2-D sagittaler Rekonstruktion. (c) Nach 12 Monaten. Kein Nachweis einer avaskulären Kopfnekrose. Unten: konservativ behandelte OTA Type 11-B2-Fraktur (extrakapsulär). (a) Konventionelle Aufnahme vor Reposition. (b) Nach geschlossener Reposition. (c) Nach 12 Monaten Nachweis einer Varusfehlstellung, erstmals festgestellt nach 8 Wochen (aus [3]).

Tab. 2 Zusammenfassung der Studie von Olerud et al. [12] (aus [8]).

\begin{tabular}{|c|c|c|c|}
\hline & $\begin{array}{l}\text { winkelstabile } \\
\text { Platte (Philos) }\end{array}$ & $\begin{array}{l}\text { konservative } \\
\text { Behandlung }\end{array}$ & Differenz \\
\hline randomisiert & 30 & 30 & \\
\hline verstorben & 2 & 2 & \\
\hline nachuntersucht nach 2 Jahren & 27 & 26 & \\
\hline anatomisch & $86 \%$ & $14 \%$ & \\
\hline Korrekturverlust & $23 \%$ & & \\
\hline Mean Flexion & $120^{\circ}$ & $111^{\circ}$ & $9^{\circ}, p=0,36$ \\
\hline Mean Abduction & $114^{\circ}$ & $106^{\circ}$ & $8^{\circ}, p=0,28$ \\
\hline Constant Score & 61 & 58 & $3, p=0,64$ \\
\hline DASH Score & 26 & 35 & $9, p=0,19$ \\
\hline HRQoL (EQ-5D) & 0,70 & 0,59 & $0,11, p=0,26$ \\
\hline $\begin{array}{l}\text { Komplikation mit größeren ungeplan- } \\
\text { ten Operationen wegen Infektion, } \\
\text { Pseudarthrose, Kopfnekrose }\end{array}$ & $n=4(13 \%)$ & & \\
\hline $\begin{array}{l}\text { Komplikation mit kleineren ungeplan- } \\
\text { ten Operationen wegen Schraubenper- } \\
\text { foration, Gelenksteife, Impingement }\end{array}$ & $\mathrm{n}=5(17 \%)$ & & \\
\hline Summe ungeplanter Operationen & $n=9(30 \%)$ & & \\
\hline Pseudarthrose & 1 & 1 & \\
\hline
\end{tabular}

In einer weiteren Publikation basierend auf dem gleichen Patientengut untersuchte die Arbeitsgruppe die gesundheitsbezogene Lebensqualität mithilfe des 15D-Instruments (Tab. 1). Dabei werden 15 allgemeine Aspekte (15 dimensions oder abgekürzt 15D) des täglichen Lebens (bewegen, sehen, hören, Alltagstätigkeiten, Vitalität etc.) abgefragt und mit Werten zwischen 0 (Tod) und 1 (perfekte Gesundheit) skaliert (http://www.15D-instrument.net). $\mathrm{Zu}$ allen Messpunkten (0, 3, 6, 12 Monate) war die mit dem 15D-Instrument gemessene gesundheitsbezogene Lebensqualität ohne statistisch signifikante Unterschiede zwischen den beiden Gruppen. Der Unterschied der mittleren direkten und indirekten Behandlungskosten der beiden Behandlungsgruppen war ebenfalls ohne statistisch signifikante Unterschiede und betrug $23953 €$ in der operierten Gruppe gegenüber $21878 €$ in der konservativen Gruppe [4].

Beide Studien haben Einschränkungen. So konnte nicht in allen Fällen eine anatomische Reposition erzielt werden. Die verwendete winkelstabile Platte war nicht anatomisch vorgeformt, wie sie heute weitgehend verwendet wird. Eine relativ niedrige Rate an Pull-out $(n=1)$ verglichen mit anderen Studien macht es aber unwahrscheinlich, dass die Ursache für die limitierten Ergebnisse im verwendeten Implantatdesign der operativen Gruppe liegt [4].

\section{Olerud-Studie: dislozierte 3-Teile- Frakturen - OP vs. konservativ}

In 2011 wurde noch eine 2. prospektiv randomisierte Studie (operativ versus konservativ) veröffentlicht mit der Besonderheit eines Nachuntersuchungszeitraums von 2 Jahren. Eingeschlossen wurden 60 Patienten mit dislozierten 3Teile-Frakturen mit einem Durchschnittsalter von 74 Jahren (56-92) (Tab. 2) [12].

In dieser Studie scheinen die operativ versorgten Fälle tendenziell etwas bessere Funktion (Beweglichkeit, Scores) zu haben, jedoch waren die Unterschiede statistisch nicht signifikant. Erheblich war der Unterschied in der Rate der ungeplanten Operationen, die in der operativen Gruppe mit 30\% signifikant erhöht war. Bei nur 7\% der Patienten wurde die Reoperation im 1. Jahr durchgeführt, bei weiteren $23 \%$ erfolgte der Eingriff erst im 2. Jahr. Es zeigte sich auch, dass im 
Tab. 3 Zusammenfassung der Studie von Olerud et al. [11] (aus [8]).

\begin{tabular}{llcl} 
Ergebnisse nach 2 Jahren & Prothese & $\begin{array}{l}\text { konservative } \\
\text { Behandlung }\end{array}$ & P \\
\hline randomisiert & 27 & 28 & \\
\hline verstorben 2 Jahre Nachuntersuchung & 24 & 25 & \\
\hline ungeplante OP's & $11 \%$ & $4 \%$ & 0,81 \\
\hline Constant Score (0-100) & 48,3 & 49,6 & 0,36 \\
\hline Schmerz (0-15) & $12, .1$ & 11,0 & 0,57 \\
\hline Aktivitäten des täglichen Lebens (0-20) & 12,9 & 13,8 & 0,89 \\
\hline Beweglichkeit (0-40) & 20,2 & 20,1 & 0,45 \\
\hline Kraft (0-25) & 3,1 & 4,7 & 0,25
\end{tabular}

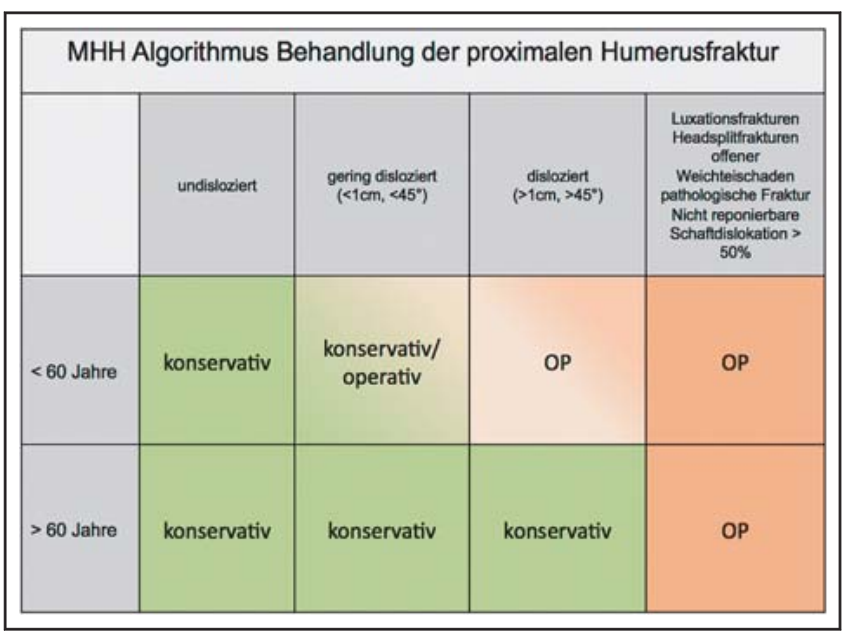

2. Jahr noch eine Verbesserung der Beweglichkeit erfolgt, vor allem bei den $\mathrm{Pa}$ tienten, die sich einem Reeingriff unterzogen haben [12].

\section{Olerud-Studie: dislozierte 3/4-Teile- Frakturen - Prothese vs. konservativ}

2011 wurde erstmals eine prospektiv randomisierte Studie mit einem Vergleich Frakturendoprothese versus konservative Therapie publiziert [11]. $55 \mathrm{~Pa}$ tienten mit dislozierten 4-Part-Frakturen und einem Durchschnittsalter von 77 Jahren (58-92) wurden randomisiert und nach 4, 12 und 24 Monaten nachuntersucht. Es fanden sich nach 24 Monaten keine signifikanten Unterschiede im Constant Score, Beweglichkeit, DASH Score und Schmerzen (VAS). Lediglich die Lebensqualität in Form des EQ-5D zeigte bessere Ergebnisse $(0,81$ versus $0,65(\mathrm{p}=0,02)$ zugunsten der Frakturprothese [11].

\section{Evidenzbasierte Therapie - welcher Patient sollte operiert werden?}

Patient > 60 Jahre

Basierend auf den neuen Evidenzniveau1-Studien haben wir einen Algorithmus entwickelt, der in Abb. 3 und 4 dargestellt ist [8]. Klare Indikationen zum konservativen Vorgehen sind alle dislozierten $\left(>1 \mathrm{~cm},>45^{\circ}\right)$ und wenig dis-



Abb. 4 MHH-Algorithmus für die konservative Behandlung proximaler Humerusfrakturen. Bei Schaftdislokation $>50 \%$ erfolgt eine geschlossene Reposition in Narkose. Bei Varusabkippung kann eine Abduktionsschiebe angelegt werden. Bei den übrigen Frakturen Gilchrist-Verband/Schultertasche für 3 Wochen (aus [8]). 


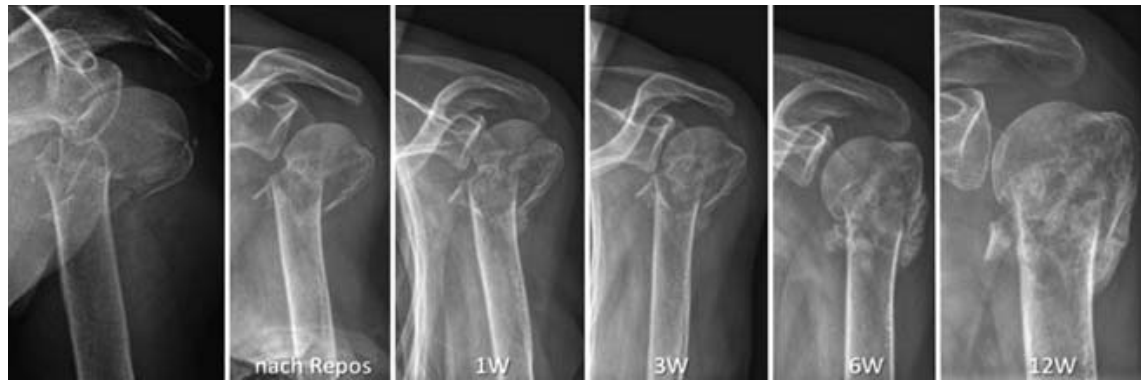

Abb. 5 Beispiel für das Potenzial zur schwerkraftinduzierten Schafttranslationskorrektur nach Repositionsmanöver unter Analgesie und proximale Rolle (aus [8]).

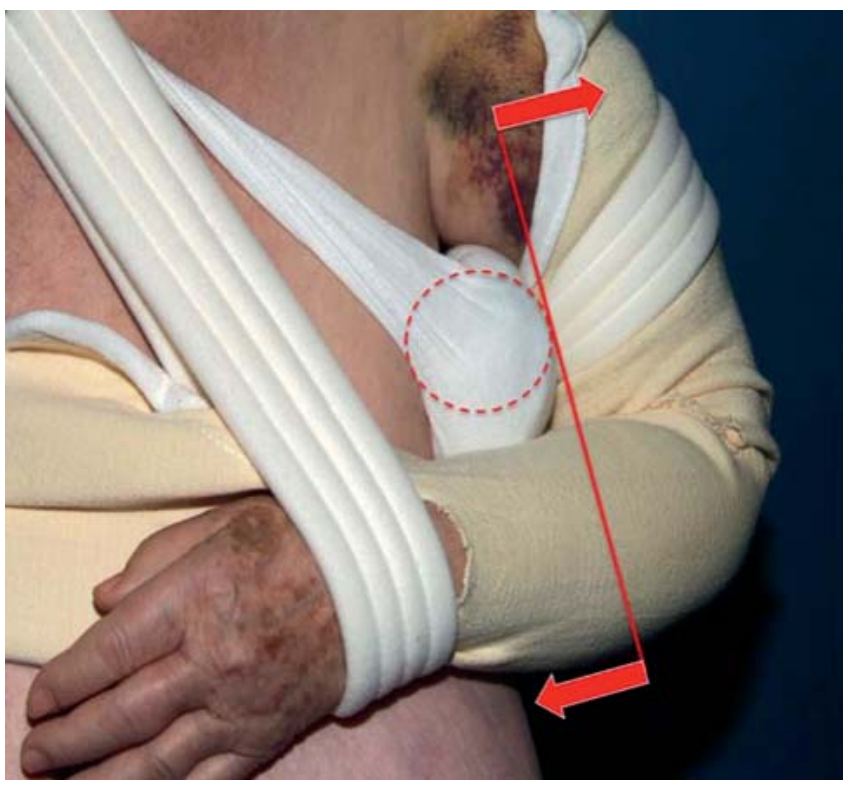

Abb. 6 Biomechanisches Prinzip der proximalen Rolle bei Schafttranslation. Durch die Schwerkraft des Armes kommt es über das Hypomochlion der Rolle (z. B. elastische Bindenrolle) zu einer Translation des proximalen Endes des distalen Hauptfragments nach lateral (aus [8]).

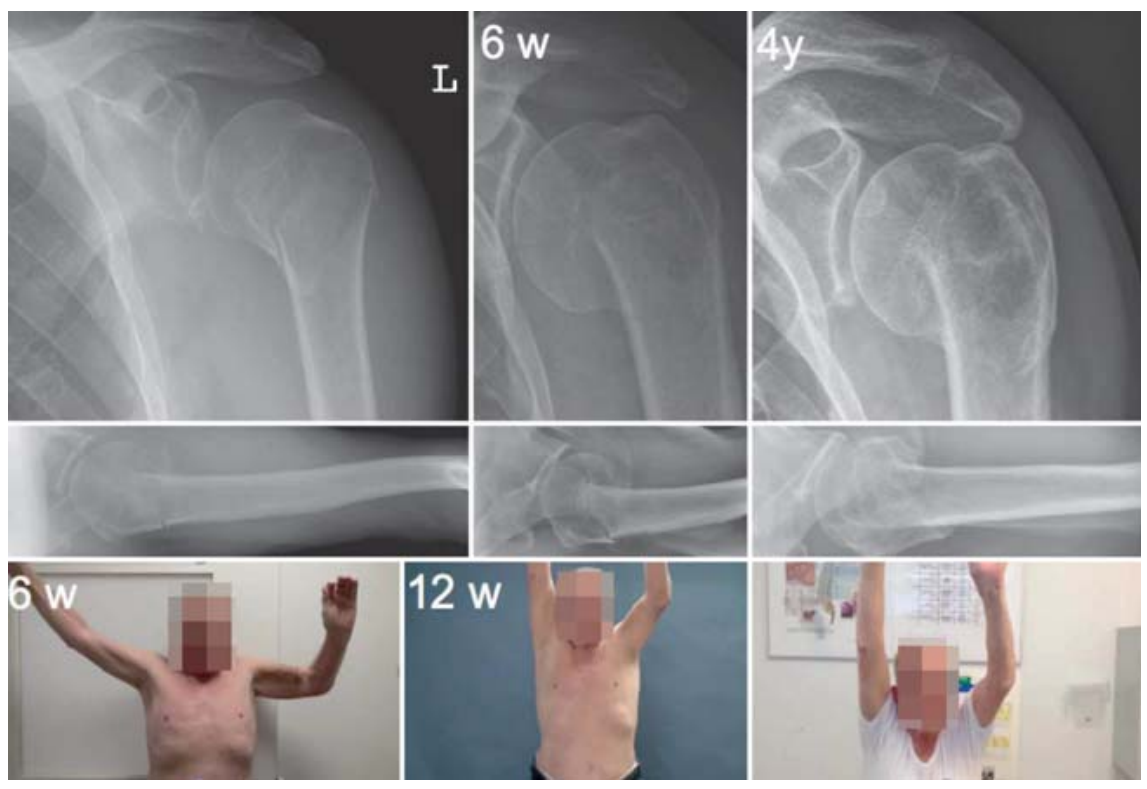

Abb. 7 84-jähriger Patient, B2-Fraktur. Trotz dislozierter Fraktur mit hochstehendem Tuberculum majus schmerzfreie gute Funktion (Constant Score 83). lozierten Frakturen $\left(<1 \mathrm{~cm},<45^{\circ}\right)$ bei Patienten $>60$ Jahre, sofern sich keine zwingenden OP-Indikationen ergeben (Luxationsfrakturen, Headsplit-Frakturen, offener Weichteilschaden, Frakturen mit neurovaskulären Verletzungen, pathologische Frakturen und nicht geschlossen reponierbare Schaftdislokation $>50 \%$ ). Grundsätzlich müssen aber immer auch die patientenbezogenen Faktoren (Alter, Komorbidität, Aktivitätsanspruch, Begleitverletzungen) mit einbezogen werden. Schaftdislokation $>50 \%$ werden in Analgesie/Kurznarkose reponiert. Dieses Vorgehen ist durch die Literatur ausreichend belegt [3].

\section{Patient < 60 Jahre}

Beim Patienten unter 60 Jahren ist die Evidenzlage erheblich schlechter. Hier wird die Indikation zur OP deutlich aggressiver gestellt. Dislozierte Frakturen $\left(>1 \mathrm{~cm} \quad\right.$ [>0,5 cm Tuberkulum], >45 $\left.{ }^{\circ}\right)$ werden operiert, undislozierte Frakturen werden konservativ behandelt.

Die Schwierigkeit stellen die wenig dislozierten Frakturen $\left(<1 \mathrm{~cm},<45^{\circ}\right)$ dar, wo wir die OP-Indikation individuell stellen und die Problematik mit dem Patienten besonders intensiv diskutieren. Die Stabilitätsuntersuchung mithilfe des Röntgenbildverstärkers bringt keine Hilfestellung bei der Entscheidung für oder gegen operatives Vorgehen.

Eine schlüssige Erklärung für das generell schlechte Abschneiden der operativen Therapie ungeachtet des eingesetzten Verfahrens steht noch aus. Eine Möglichkeit könnte der im Alter oft größere subakromiale Raum und geringere Muskeltonus sein, was mehr Fehlstellung tolerieren ließe, als das beim muskelkräftigen Patienten mit engem Subakromialraum der Fall ist. Die beim operativen Vorgehen verfahrensabhängig mehr oder weniger starke Störung der Weichteile und Gleitschichten könnte ein weiterer Faktor sein.

\section{Technik der konservativen Behandlung}

Der MHH-Algorithmus für die konservative Behandlung proximaler Humerusfrakturen ist in Abb. 3 und $\mathbf{4}$ abgebildet. Die konservative Behandlung lässt sich in der Regel auch ambulant gut durchführen. Bei medialer Schaftdislokation $>50 \%$ erfolgt eine geschlossene Reposition unter Analgesie oder Narkose [3], bei geringerer Medialisierung wird proximal eine kleine Rolle (elastische 


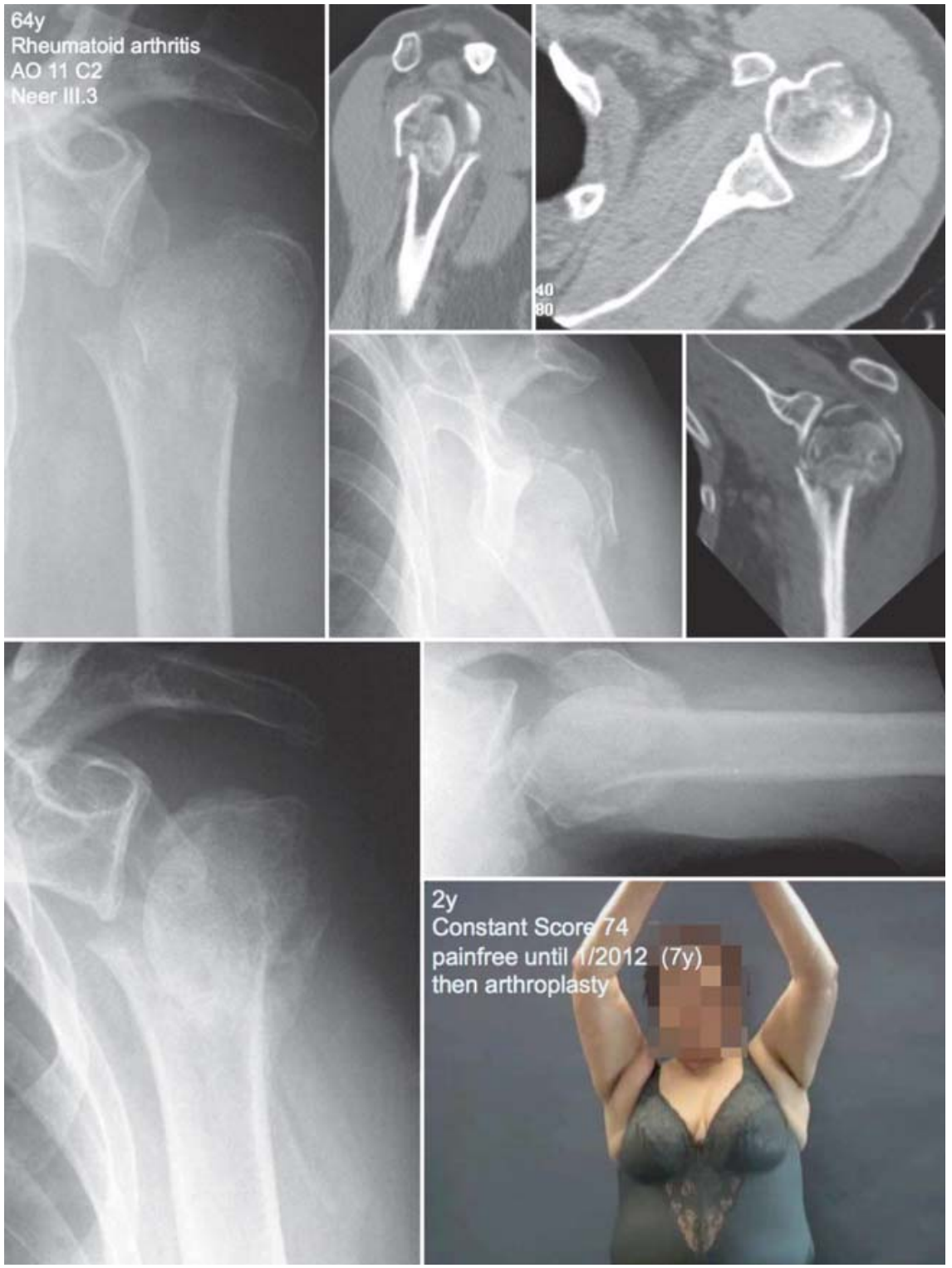

Abb. 8 64-jährige Patientin mit rheumatoider Arthritis und C2-Fraktur in 2005. Konservative Behandlung. Unten rechts die Funktion bei der 2-Jahres-Nachuntersuchung. Schmerzfrei bis 1/ 2012 (7 Jahre) (Constant Score 74), dann Auftreten von progredientem Schmerz. Im Sommer 2012 problemlose Implantation einer Schulterprothese mit guter postoperativer Funktion.

Binde) in die Achsel eingelegt. Bei Varusabkippung kann eine Abduktionsschiene sinnvoll sein.

\section{1.-3. Woche}

Bei den übrigen Frakturen Gilchrist-Verband/Schultertasche für 3 Wochen, ein Einschnüren in der Ellbeuge sollte unbedingt vermieden werden, die Hand bleibt frei. Zur Körperpflege kann der Arm herausgenommen werden, Streckübungen im Ellbogen werden als angenehm empfunden. Nach der 1 . Woche geben routinemäßig keine Schmerzmedikation. Keine Krankengymnastik, keine Eigenübungen. die der Patient in Eigenbeübung mehrfach täglich selbst durchführen soll.

Durch Einwirkung der Schwerkraft besteht ein hohes Spontankorrekturpotenzial auch bei Schafttransplation (Abb. 5), das es zu nutzen gilt. Über ein Hypomochlion ("hohe Rolle“) in Form einer z. B. elastischen Bindenrolle kann ein zusätzliches korrigierendes Moment ausgeübt werden (Abb.6).

Die Fallbeispiele in den Abb. 7-9 belegen das große Potenzial konservativer Behandlung.

\section{Zusammenfassung und Schlussfolgerungen}

Obwohl die proximale Humerusfraktur eine der häufigsten Frakturen überhaupt ist, ist die Evidenzlage schlecht. Sie hat sich aber in den letzten Jahren insbesondere durch die 3 prospektiv randomisierten Studien aus Skandinavien verbessert.

Die winkelstabile Plattenosteosynthese und intramedulläre Stabilisierung haben die Erwartungen bislang nicht erfüllen können. Bei hohen Komplikations- und Revisionsraten konnte keine Verbesserung gegenüber der konservativen Therapie erreicht werden.

Basierend auf der aktuellen Literatur ergibt sich heute ein ernüchterndes Bild. Trotz massiven Bemühungen der Industrie und trotz des Einsatzes „moderner“ Implantate mit immer noch mehr Verriegelungsmöglichkeiten liegt für die dislozierten proximalen Humerusfrakturen des älteren Patienten über 60 Jahre kein Nachweis von Vorteilen der operativen Therapie vor, der die hohen Komplikations- und Revisionsraten rechtfertigen würde.

\section{3.-6. Woche}

Durchführung von schmerzadaptierten Pendelübungen und geführte Bewegungen bis $90^{\circ}$. Verwendung der Schultertasche nach subjektivem Empfinden. Keine Krankengymnastik.

\section{6.-12. Woche}

Weglassen der Schultertasche. Durchführung schmerzadaptierter Dehnungsübungen in Eigenbeübung, unterstützt durch Krankengymnastik. Stab und Umlenkrolle sind wichtige Übungsgeräte. Demonstration von Stretchingübungen,
Operative und konservative Verfahren dislozierten Frakturen des Patienten über 60 Jahre ähnlich gut oder schlecht. Dieses nicht unterschiedliche Ergebnis ist aber im Fall der winkelstabilen Plattenosteosynthesen mit einer statistisch signifikant höheren Komplikationsrate behaftet, die in den meisten Studien um $30 \%(20-50 \%)$ liegt [8]. Die aktuell vorliegenden Daten rechtfertigen die zurzeit äußerst großzügig gestellten OP-Indikationen beim älteren Patienten nicht. Der Satz ,... If it looks normal, it works normal ..." bestätigt sich aus unserer Sicht am proximalen Humerus nicht. sind im funktionellen Ergebnis auch bei 


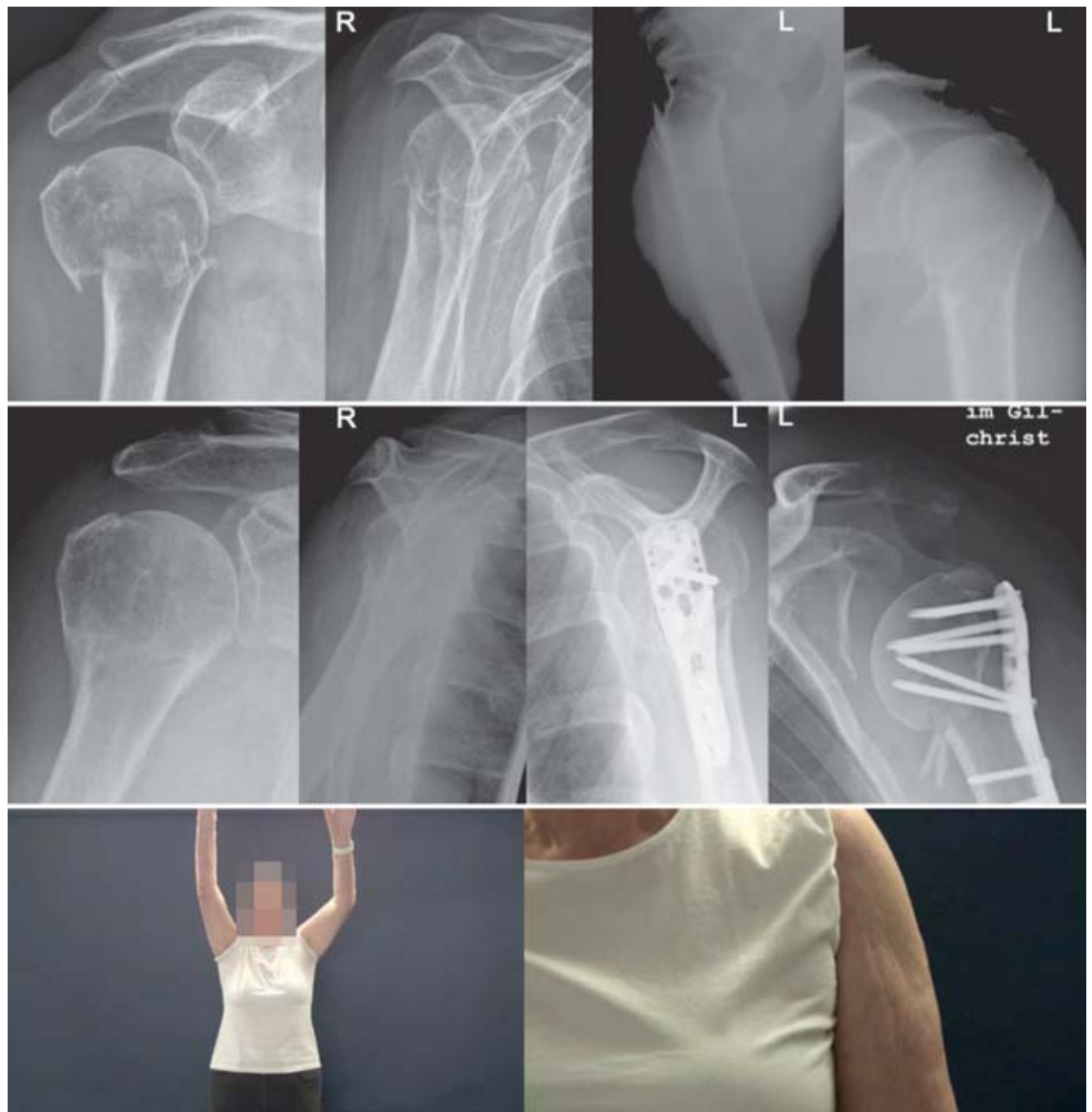

Abb.9 Oben: 76-jährige Patientin, frische B1-Fraktur rechts. Auf der linken Gegenseite ähnliche Fraktur, die auswärts operativ mit winkelstabiler Platte stabilisiert wurde. Mitte: Frakturen im Verlauf. Ausheilungsbilder des rechten Humerus. Verlaufsbild der opertierten linken Seite mit Gilchristverband. Unten: beidseits schmerzfreie gute Funktion, auf der rechten, konservativ behandelten Seite sogar besser beweglich (Constant Score 78) als links (Constant Score 75).

Zwingende OP-Indikationen in allen Alterstufen sind Luxations- und HeadsplitFrakturen, offene Frakturen, manche Frakturen mit neurovaskulärem Schaden, die meisten pathologischen Frakturen und die nicht reponierbare Schaftdislokation $>50 \%$ Schaftbreite.

Beim Patienten unter 60 Jahren sollten dislozierte Frakturen operiert werden. Undislozierte Frakturen werden konservativ behandelt. Die Schwierigkeit stellen die wenig dislozierten Frakturen dar, wo die OP-Indikation individuell gestellt und die Problematik mit dem Patienten besonders intensiv diskutiert werden sollte. Die Stabilitätsuntersuchung mithilfe des Röntgenbildverstärkers bringt keine Hilfestellung bei der Entscheidung für oder gegen operatives Vorgehen. Gerade für den unter 60-jährigen Patienten brauchen wir mehr und bessere Daten. Hier muss verstärkt daran gearbeitet werden, gute Evidenz in Form von sorgfältig geplanten (Fallzahl, Nachunter- suchungszeitraum) prospektiv randomisierten Studien zu generieren.

Konventionelle oder inverse Prothesen sind komplexen, nicht rekonstruierbaren Frakturformen vorbehalten. Auch für die dislozierte 4-Teile-Fraktur des über 60jährigen Patienten ist der statistisch haltbare Nachweis der Überlegenheit der Frakturprothese in der prospektiv randomisierten Analyse noch nicht gelungen.

\section{Literatur}

${ }^{1}$ Acklin YPx, Jenni R, Walliser $M$ et al. Minimal invasive PHILOS ${ }^{\circledR}$-Plate Osteosynthesis in proximal humeral fractures. Eur J Trauma 2008; 35: 35-39

2 Bogner R, Hübner C, Matis N et al. Minimallyinvasive treatment of three- and four-part fractures of the proximal humerus in elderly patients. J Bone Joint Surg $[\mathrm{Br}] 2008 ; 90$ : 1602-1607 treatment with an angular stable plate for complex displaced proximal humeral fractures in elderly patients: a randomized con-
${ }^{3}$ Fjalestad T, Hole MØ, Hovden IA et al. Surgical trolled trial. J Orthop Trauma 2011; 26: 98 106

${ }^{4}$ Fjalestad T, Hole MO, Jorgensen JJ et al. Health and cost consequences of surgical versus conservative treatment for a comminuted proximal humeral fracture in elderly patients. Injury 2010; 41: 599-605

5 Gaebler C. McOueen MM, Court-Brown CM. Minimally displaced proximal humeral fractures: epidemiology and outcome in 507 cases. Acta Orthop Scand 2003; 74: 580-585

${ }^{6}$ Hertel $R$. Fractures of the proximal humerus in osteoporotic bone. Osteoporosis Int 2005; 16 (Suppl. 2): S65-S72

${ }^{7}$ Krettek C. "If It Looks Normal, It Works Normally" Is Challenged in Proximal Humeral Fractures. J Bone Joint Surg [Am] 2012; 94: 1

${ }^{8}$ Krettek C, Wiebking U. [Proximal humerus fracture: is fixed-angle plate osteosynthesis superior to conservative treatment?]. Unfallchirurg 2011; 114: 1059-1067

${ }^{9}$ Kristiansen B, Kofoed H. Transcutaneous reduction and external fixation of displaced fractures of the proximal humerus. A controlled clinical trial. J Bone Joint Surg $[\mathrm{Br}]$ 1988; 70: 821-824

${ }^{10}$ Nho SJ, Brophy RH, Barker JU et al. Management of proximal humeral fractures based on current literature. J Bone Joint Surg [Am] 2007; 89 (Suppl. 3): 44-58

11 Olerud P Ahrengart L Ponzer S et al. Hemiarthroplasty versus nonoperative treatment of displaced 4-part proximal humeral fractures in elderly patients: a randomized controlled trial. J Shoulder Elbow Surg 2011; 20: 10251033

12 Olerud P, Ahrengart L, Ponzer S et al. Internal fixation versus nonoperative treatment of displaced 3-part proximal humeral fractures in elderly patients: a randomized controlled trial. J Shoulder Elbow Surg 2011; 20: 747755

${ }^{13}$ Resch H, Povacz P, Frohlich R et al. Percutaneous fixation of three- and four-part fractures of the proximal humerus. J Bone Joint Surg [Br] 1997; 79: 295-300

${ }^{14}$ Ring D. Current concepts in plate and screw fixation of osteoporotic proximal humerus fractures. Injury 2007; 38: (Suppl.3): S59S68

15 Sommer C, Gautier E, Müller M et al. First clinical results of the Locking Compression Plate (LCP). Injury 2003; 34 (Suppl. 2): B43-B54

${ }_{16}$ Wall B, Walch G. Reverse shoulder arthroplasty for the treatment of proximal humeral fractures. Hand Clin 2007; 23: 425-430

\section{Prof. Dr. med. Christian Krettek,} FRACS, FRCSEd

Arzt für Orthopädie und Unfallchirurgie

Arzt für Chirurgie/Unfallchirurgie Direktor

\section{Dr. med. Nael Hawi}

Assistenzarzt

\section{Dr. med. Ulrich Wiebking}

Assistenzarzt

Unfallchirurgische Klinik

Medizinische Hochschule Hannover (MHH)

Carl-Neuberg-Straße 1

30625 Hannover

Krettek.Christian@mh-hannover.de 